\title{
Thèses remarquées
}

\author{
Développement d'une forme pharmaceutique véhiculant un calixarène destinée au \\ traitement d'une peau saine ou lésée contaminée par de l'uranium, Aurélie \\ Spagnul. Thèse de l'université Paris-XI, soutenue par le 16 novembre 2009. \\ Laboratoires d'accueil : Laboratoire de Radiochimie, IRSN/DRPH/SDI, Fontenay- \\ aux-Roses, Laboratoire de Physico-chimie, Pharmacotechnie et Biopharmacie, \\ Chatenay-Malabry, encadrants IRSN : Dr Céline Bouvier-Capely et Dr Guillaume \\ Phan, directeur de thèse : Pr. Elias Fattal.
}

Les accidents de contamination par voie cutanée représentent la seconde cause de contamination des travailleurs de l'industrie nucléaire, après l'inhalation. La contamination cutanée par l'uranium est particulièrement préoccupante car elle peut conduire à une forte exposition interne des individus et les traitements actuellement employés, c'est-à-dire le rinçage de la zone cutanée contaminée et l'administration de décorporants, sont peu efficaces. Il apparaît alors important de développer de nouveaux traitements. La stratégie adoptée dans ces travaux de thèse a consisté à mettre au point un traitement local de la contamination cutanée par l'uranium basé sur l'utilisation d'un chélateur de la famille des calixarènes, le 1,3,5- $\mathrm{OCH}_{3}-2,4,6-\mathrm{OCH}_{2} \mathrm{COOH}-p$ tertbutylcalix[6]arène, permettant de piéger le radioélément au niveau de la zone cutanée contaminée afin d'empêcher sa pénétration dans l'organisme et ainsi d'éviter la manifestation de ses effets toxiques.

Notre premier objectif a été de développer une forme galénique adaptée à l'usage cutané contenant le calixarène tricarboxylique d'intérêt et capable de le rendre disponible pour la chélation de l'uranium présent dans une solution aqueuse contaminée. Une nanoémulsion de type huile dans eau caractérisée par la présence de molécules de calixarène à la surface des gouttelettes huileuses dispersées a ainsi été formulée.

Notre second objectif a consisté à évaluer l'intérêt de la nanoémulsion de calixarène en tant que traitement d'urgence des contaminations cutanées par l'uranium. Dans un premier temps, nous avons démontré par des expérimentations in vitro utilisant la technique d'ultrafiltration que la nanoémulsion de calixarene est capable d'extraire rapidement les ions uranyle d'une solution aqueuse contaminée. L'étude ex vivo de la diffusion percutanée de l'uranium à travers des explants de peau d'oreille de porc montés en cellule de Franz a ensuite montré que l'application de la nanoémulsion de calixarène immédiatement après la contamination cutanée permet d'empêcher le passage de l'uranium à travers la peau intacte et la peau excoriée.

Nous avons donc mis au point au cours de cette thèse une forme galénique véhiculant un chélateur original de l'uranium. Ce système montre de réelles potentialités et permet d'envisager de nouvelles perspectives dans le traitement des contaminations cutanées par l'uranium. 
Reconstruction 3D complète par modélisation Monte Carlo de la matrice système. Apport aux approches quantitatives à l'iode-131, Thomas Carlier. Thèse de l'université de Nantes, soutenue en décembre 2009, Centre de Recherche en Cancérologie, directeur de thèse : Jacques Barbet, DR CNRS, co-directeur de thèse : Françoise Kraeber-Bodéré, PU-PH .

La correction des phénomènes physiques dégradant l'information quantitative en tomographie par émission monophotonique (SPECT) a fait l'objet de très nombreux développements au cours de cette dernière décennie. L'utilisation de $\mathrm{l}^{131} \mathrm{I}$ complique singulièrement les méthodes de correction à mettre en œuvre compte tenu de son spectre d'émission incluant des émissions de hautes énergies et des nombreux biais qui en découlent.

Une solution très générale à la problématique de reconstruction tomographique incluant sans hypothèses contraignantes les corrections d'atténuation, de diffusion et de réponse non stationnaire du détecteur a été proposé par Floyd en 1986 et consiste à calculer entièrement la matrice système, utilisée dans un algorithme de reconstruction itératif, par une méthode Monte Carlo. Cette idée a récemment était étudiée par Lazaro en 2005 et a montré des résultats particulièrement encourageant pour des acquisitions au ${ }^{99 \mathrm{~m}} \mathrm{Tc}$.

L'objectif de ce travail de thèse a été de mettre en œuvre cette méthode de reconstruction tridimensionnelle complète par modélisation Monte Carlo de la matrice système (F3DMC) et de l'optimiser en vue de son utilisation dans un contexte clinique.

Les premiers développements se sont portés sur le calcul de la matrice système relative à un fantôme simple de type Jaszczak rempli uniformément auquel deux sphères homogènes ont été rajoutées. Compte tenu du nombre important de particules à simuler pour obtenir un projecteur statistiquement robuste, les calculs ont nécessité l'utilisation de l'infrastructure de calcul et de stockage massif EGEE. Les projections acquises et simulées ont été reconstruites en montrant d'une part une bonne précision de la restauration de l'activité dans le bruit de fond et dans des sphères par rapport à des méthodes plus conventionnelles et d'autre part la nécessité de construire un projecteur statistiquement plus robuste.

À cette fin, nous avons utilisé une nouvelle technique d'accélération des simulations fondée sur la tabulation de la fonction de réponse angulaire du couple collimateur/détecteur (ARF). Elle repose sur le calcul préalable d'une fonction dépendante de l'énergie incidente du photon, de sa position d'interaction relative à sa position d'émission, de la fenêtre spectrométrique de détection et de la résolution en énergie du détecteur. Nous avons validé l'approche ARF dans différents cas en allant du plus simple (point ou plan source dans l'air) au plus réaliste (milieu de propagation hétérogène sur la base d'acquisition tomodensitométrique). À qualité de signal équivalent et en comparaison avec une simulation GATE standard nous avons mis en évidence un gain maximal de 140 pour un milieu de propagation composé uniquement d'air et proche de 100 lorsqu'un milieu diffusant hétérogène est considéré. L'application immédiate qui a pu être déduite de ces ordres de grandeurs est la possibilité de réaliser une simulation SPECT à ${ }^{1131}$ I réaliste d'un patient en 2 jours maximum sous réserve de la disponibilité d'une petite grappe de calcul composée d'environ 20 CPUs. Dans le cadre de notre problématique de reconstruction F3DMC, nous pouvions ainsi espérer réduire très significativement les biais quantitatifs dans le volume reconstruit dus au bruit présent dans la matrice système. 


\section{THÈSES REMARQUÉES}

Nous avons ainsi étendu la méthodologie ARF au calcul de la matrice système dans GATE. L'impact de cette modification a été étudié au moyen de la construction de deux matrices systèmes dont la première correspondait au fantôme analytique déjà étudié précédemment et la deuxième à un cas réaliste sur la base de l'acquisition tomographique d'un patient. Nous avons montré sur les données simulées une nette amélioration des paramètres quantitatifs avec une réduction du bruit dans les images reconstruites par rapport à une reconstruction F3DMC sans utilisation de l'ARF. La méthodologie ARF rend donc possible le calcul Monte Carlo d'une matrice système dans un temps raisonnable avec un gain important en termes de précision de quantification, de contraste et de bruit dans les données reconstruites. L'application de la reconstruction F3DMC a montré une bonne restauration de l'activité dans différentes zones d'intérêts avec des écarts inférieurs à $7 \%$ lorsque nous nous sommes intéressés aux projections simulées d'un patient. Les reconstructions des données acquises de ce même patient ont donné des résultats relativement proches de la reconstruction utilisée en routine clinique sans que nous puissions toutefois juger complètement de la pertinence de ces résultats étant donné, d'une part, que nous ne connaissons pas la distribution réelle d'activité volumique et, d'autre part, que nous avons mis en évidence des imperfections lors des reconstructions réalisées sur les projections acquises du fantôme analytique. 